Repository of the Max Delbrück Center for Molecular Medicine (MDC) Berlin (Germany)

\title{
CCN1 mutation is associated with atrial septal defect
}

Perrot, A., Schmitt, K.R., Roth, E.M., Stiller, B., Posch, M.G., Browne, E.N.L., Timmann, C., Horstmann, R.D., Berger, F., Ozcelik, C. 


\title{
CCN1 mutation is associated with atrial septal defect
}

\author{
Andreas Perrot ${ }^{1 *}$, Katharina R. Schmitt ${ }^{2}$, Eva-Maria G. Roth ${ }^{2}$, Brigitte Stiller ${ }^{3}$, Maximilian G. Posch ${ }^{1,4}$, \\ Edmund N.L. Browne ${ }^{5}$, Christian Timmann' ${ }^{6}$ Rolf D. Horstmann ${ }^{6}$, Felix Berger ${ }^{2,7}$, Cemil Özcelik ${ }^{1,8}$
}

${ }^{1}$ Charité-Universitätsmedizin Berlin, Experimental \& Clinical Research Center, a joint cooperation between the Charité Medical Faculty and the Max-Delbrück Center for Molecular Medicine, Berlin, Germany

${ }^{2}$ Department of Congenital Heart Disease and Pediatric Cardiology, German Heart Institute Berlin, Berlin, Germany

${ }^{3}$ Department of Congenital Heart Disease, University Heart Center Freiburg-Bad Krozingen, Freiburg, Germany

${ }^{4}$ Charité Research Organisation GmbH, Berlin, Germany

${ }^{5}$ Department of Community Health, School of Medical Sciences, Kwame Nkrumah University of Science and Technology, Kumasi, Ghana

${ }^{6}$ Department of Molecular Medicine, Bernhard Nocht Institute for Tropical Medicine, Hamburg, Germany

${ }^{7}$ Otto Heubner Centrum at Campus Virchow-Klinikum, Charité-Universitätsmedizin Berlin, Berlin, Germany

${ }^{8}$ Helios Albert-Schweitzer-Klinik, Northeim, Germany

\section{*Corresponding author:}

Andreas Perrot, MSc

Cardiovascular Genetics

Experimental \& Clinical Research Center (ECRC)

Charité-Universitätsmedizin Berlin

Lindenberger Weg 80, 13125 Berlin

Tel: +4930450540168

Email: andreas.perrot@charite.de 


\begin{abstract}
The genetic basis of congenital heart disease remains unknown in most of the cases. Recently, a novel mouse model shed new light on the role of CCN1/CYR61, a matricellular regulatory factor, in cardiac morphogenesis. In a candidate gene approach, we analyzed a cohort of 143 patients with atrial septal defects (ASD) by sequencing the coding exons of CCN1. In addition to three frequent polymorphisms, we identified an extremely rare novel heterozygous missense mutation $(c .139 C>T$; p.R47W) in one patient with severe ASD. The mutation leads to an exchange of residues with quite different properties in a highly conserved position of the Nterminal insulin-like growth factor binding protein (IGFBP) module. Further bioinformatic analysis, exclusion of known ASD disease genes as well as the exclusion of the mutation in a very high number of ethnically matched controls (more than 1000 individuals) and in public genetic databases indicates that the $p . R 47 \mathrm{~W}$ variant is a probable disease-associated mutation. The report about ASD in mice in heterozygous Cen1 +/- animals strongly supports this notion. Our study is the first to suggest a relationship between a probable $C C N 1$ mutation and ASD. Our purpose here was to draw attention to $C C N 1$, a gene that we believe may be important for genetic analysis in patients with congenital heart disease.
\end{abstract}

Keywords: Atrial septal defect; ASD; CCN1; Congenital heart disease 


\section{Introduction}

Congenital heart disease (CHD) is the most common birth defect in humans. The etiology for most patients is unknown. Cardiogenesis is complex and appears highly susceptible to perturbations resulting in heart malformations [1, 2]. Among the most sensitive cardiac structures are the atrial and ventricular septa. Numerous advances in understanding the molecular pathways and genes involved in septal defects have been published in the past few years [1,2]. Single gene mutations were identified as heritable risk factors for atrial septal defects (ASD) in a number of patients (as reviewed by us in Posch et al. [3]). Various mutations in transcription factors and sarcomeric genes were described in different studies [3]. We have analyzed different genes involved in the pathogenesis of CHDs and could identify mutations in ASD patients $[4,5,6]$.

The CCN protein family is a novel class of signal modulators named after three prototypical members, cysteine-rich

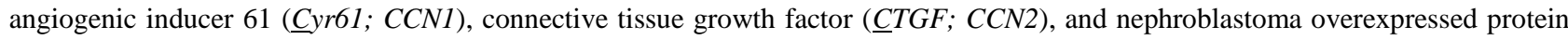
(Nov; CCN3) [7]. Matricellular CCN proteins are regulatory factors involved in cell signalling participating in various crucial biological processes like cell differentiation, adhesion, and apoptosis [7]. A mouse model shed new light on the role of CCN proteins in cardiac valvuloseptal morphogenesis: Mo and Lau described the Ccn1-null mouse showing severe septal defects with complete penetrance [8]. Therefore, CCN1 was considered a promising candidate gene for ASD in man.

\section{Materials and Methods}

We enrolled 143 patients (most of them of Caucasian origin) after informed consent was obtained. All of them underwent extensive non-invasive and invasive cardiovascular evaluation. The study cohort consisted of unrelated patients with atrial septal defect (ASD), nearly all sporadic cases. The vast majority $(\mathrm{n}=134 ; 94 \%)$ had an isolated ostium secundum atrial septal defect (ASD II). Only 9 patients $(6 \%)$ had ASD II with concomitant minor CHD (including aortic coarctation, persistent ductus arteriosus, and partial anomalous venous return). The first control group of 300 unrelated Caucasians had no CHD as shown by echocardiography. The second control group - matched to the ethnicity of the identified patient - comprised 1015 samples randomly selected from a group of 5100 unrelated adults, residents of the Ashanti region in Ghana. The study complies with the 1964 Declaration of Helsinki. The research protocol has been approved by the locally appointed ethics committees in Berlin and Kumasi and informed consent has been obtained from the enrolled subjects.

We sequenced the five exons of CCN1/CYR61 (Genbank accession number NM_001554) using PCR and ABI technology (Applied Biosystems, Darmstadt, Germany). After identification of variants, allele frequencies were assessed in controls using restriction endonuclease digestion (Ava II) or using HybProbe chemistry and a LightCycler (Roche, Mannheim, Germany). The significance of the identified variant $p \cdot R 47 \mathrm{~W}$ was assessed by considering the conservation of the affected amino acid, the nature and location of the change, its rarity assessed in own controls and population-based datasets, and the possible impact of the amino acid substitution by five different mutation prediction tools (Mutation Taster [9], MutPred [10], SIFT [11], Polyphen2 [12], and SNAP [13]). We analyzed p.R47W by checking two large population-based databases, dbSNP (release 138 at http://www.ncbi.nlm.nih.gov/snp) and the Exome Sequencing Project (ESP6500SI-V2 dataset) of the National Heart, Lung and Blood Institute (http://evs.gs.washington.edu/EVS/ [14]). 


\section{Results}

In total, we found one novel and three known variants in the CCN1 gene (Fig. 1c). Three of these variants (p.L192L, p.S316C, and p.R334W) were identified as being polymorphisms because they were found in various frequencies in both cohorts, patients and Caucasian controls, and were registered in dbSNP. In contrast, we identified the novel heterozygous $c .139 C>T$ missense variant in exon 2 (Fig. 1b) in one patient only. The mutation leads to an exchange of the positively charged residue arginine to the aromatic hydrophobic residue tryptophane at codon $47(p . R 47 W)$. We did not find this variant in dbSNP and the ESP dataset which points to the novelty and extreme rarity of $p . R 47 W$. It is located in module 1 of the CCN1 protein, namely the N-terminal insulin-like growth factor binding protein (IGFBP) domain, very closely to the "IGFBP motif" (Fig. 1a). The affected amino acid is highly conserved in orthologs of 14 vertebrates (ranging from chimp to zebrafish) and in paralogs of the human IGFBP isoforms 1-4 (Fig. 1a). While only PolyPhen 2 predicted $p . R 47 \mathrm{~W}$ as neutral, the other four mutation prediction tools predicted the variant as deleterious. The patient with the $p . R 47 W$ mutation was of West African origin. Because of the high degree of genetic variability found in Africans, we chose controls with the same ethnic origin, a cohort of 1015 unrelated individuals from West Africa. All 2030 control alleles were negative for the mutation (as well as 600 alleles from the Caucasian controls without CHD). The 4406 alleles of African American origin in the ESP dataset did also not show this mutation. Further, the disease genes GATA4 and NKX2.5 that are known to cause nonsyndromic ASD, were excluded as cause of the disease in this patient as described previously [4].

The male black patient aged 36 years was admitted to the hospital because of exertional dyspnoea. Physical examination revealed no signs of cyanosis, pulmonary congestion, or peripheral edema. The ECG confirmed sinus rhythm and showed an incomplete right bundle branch block. Furthermore, a crochetage pattern of the $\mathrm{R}$ wave was present in the inferior limb leads (Fig. 2a). The transthoracic echocardiography (TTE) revealed severe enlarged right atrium and ventricle. The function of both ventricles was maintained. A significant ASD (3.7 cm in diameter) with a large left-right shunt was demonstrated by TTE (Fig. 2b). Cardiac catheterization revealed a pulmonary artery blood flow relative to systemic blood flow (Qp/Qs) of 5:1. Since there was only a rudimental atrial septum, the finding was considered as a common atrium. The defect was surgically closed with a Gore-Tex patch. Unfortunately, we had no access to his family.

\section{Discussion}

To our knowledge, this report is the first to suggest a relationship between a genetic variant in CCN1, a growth-factor inducible immediate-early gene, and a human disease (see Online Mendelian Inheritance in Man OMIM No. *602369). Although the patient's family was not available, there are numerous findings supporting the notion that the $p . R 47 W$ variant is a probable disease-associated mutation rather than a rare polymorphism.

The extreme rarity of the mutation in the patient cohort, as also reported for the other ASD associated variants [3], and the exclusion of this variant in a very high number of ethnically well matched controls (our own 1015 controls and 2203 African Americans from the ESP dataset) are important points, as well as the exclusion of known ASD disease genes. The mutation leads to an exchange of residues with quite different properties in a highly conserved position of the IGFBP module harboring the important 
"IGFBP motif" found in all IGFBPs and CCN proteins [15] suggesting a disturbed protein folding (strengthened by special bioinformatic tools). This motif is the central element of the "palm-like" protein structure, around which a polypeptide chain is bent, forming a disulfide ladder and assuring a proper spatial relationship between the key IGF-binding residues in that domain [16]. Interestingly, Buckway et al. showed by site-directed mutagenesis that single missense mutations in this N-terminal domain, adjacent to codon 47, were sufficient to weaken the IGF binding of IGFBP3 which shares high homology with CCN1 [17]. Therefore, it seems highly probable that the $p \cdot R 47 \mathrm{~W}$ mutation also may alter the IGF binding of this domain, thus modulating IGF's biological effects in embryonic development. Indeed, IGFs are potent cell survival signals (in combination with IGFBPs as regulators of this function) influencing apoptosis, one of the key mechanisms for sculpting embryonic tissue [18]. Furthermore, Zhu at al. reported a previously unknown function of IGFBP-4 as a cardiogenic growth factor by knockdown of the homologous IGFBP-4 in Xenopus embryos which resulted in cardiac defects [19].

Limitations of our study are the lack of cosegregation and of biochemical data concerning an effect of the mutation. Indeed, it is most likely that the majority of patients with ASD have a complex, multifactorial aetiology [3]. But one should also note that the cardiac phenotype most frequently seen in published mutation carriers are ASDs [3]. It may be possible that the p.R47W variant is unrelated to the disease. But our genetic data and pathophysiologic conclusions in combination with the Ccnl mouse model fit together quite well and contradict this notion. Indeed, one of the strongest arguments supporting our data is the remarkable observation by Mo and Lau that haploinsuffiency for Ccnl (heterozygous +/- mice) also resulted in delayed formation of the ventricular septum in the mouse embryo and persistent ASD in $20 \%$ of adult mice [8]. In these animals, Ccn1 haploinsuffiency caused precocious apoptosis in the atrial junction of the cushion tissue and impaired gelatinase activities in the muscular component of the interventricular septum, when fusion between the endocardial cushion tissue and the atrial and ventricular septa occurred [8]. Clearly, deficiency in matrix signaling by $\mathrm{CCN}$ proteins could lead to autosomal dominant septal defects, identifying heterozygous Ccn1 mice as a genetic model for ASD.

In conclusion, using a translational approach we identified the first probable mutation $(p \cdot R 47 W)$ in a patient with large ASD linking $C C N 1$, an important factor in embryogenesis and subsequent development, to human pathology. Our results underscore close homology between the mouse model and human disease. Further studies are necessary to elucidate the mechanism of how this mutation contributes to septal defects.

Acknowledgements: The study was supported by research grants from Charité-Universitätsmedizin Berlin.

Conflict of interest: The authors declare that they have no conflict of interest. 


\section{References:}

1. Gruber PJ, Epstein JA (2004) Development gone awry: Congenital heart disease. Circ Res 94:273-283

2. Ransom J, Svivastava D (2007) The genetics of cardiac birth defects. Semin Cell Dev Biol 18:132-139

3. Posch MG, Perrot A, Berger F, Özcelik C (2010) Molecular genetics of congenital atrial septal defects. Clin Res Cardiol 99:137-147

4. Posch MG, Perrot A, Schmitt K, Mittelhaus S, Esenwein EM, Stiller B, Geier C, Dietz R, Gessner R, Özcelik C, Berger F (2008) Mutations in GATA4, NKX2.5, CRELD1, and BMP4 are infrequently found in patients with congenital cardiac septal defects. Am J Med Genet A 146A:251-3 5. Posch MG, Gramlich M, Sunde M, Schmitt KR, Lee SH, Richter S, Kersten A, Perrot A, Panek AN, Al Khatib IH, Nemer G, Mégarbané A, Dietz R, Stiller B, Berger F, Harvey RP, Ozcelik C (2010) A gain-of-function TBX20 mutation causes congenital atrial septal defects, patent foramen ovale and cardiac valve defects. J Med Genet 47:230-5

6. Posch MG, Waldmuller S, Müller M, Scheffold T, Fournier D, Andrade-Navarro MA, De Geeter B, Guillaumont S, Dauphin C, Yousseff D, Schmitt KR, Perrot A, Berger F, Hetzer R, Bouvagnet P, Özcelik C (2011) Cardiac alpha-myosin (MYH6) is the predominant sarcomeric disease gene for familial atrial septal defects PLoS One 6:e28872.

7. Leask A, Abraham DJ (2006) All in the CCN family: essential matricellular signaling modulators emerge from the bunker. J Cell Sci 119:48034810 .

8. Mo FE, Lau LF (2006) The matricellular protein CCN1 is essential for cardiac development. Circ Res 99:961-969

9. Schwarz JM, Rödelsperger C, Schuelke M, Seelow D (2010) MutationTaster evaluates disease-causing potential of sequence alterations. Nat Methods 7:575-6

10. Li B, Krishnan VG, Mort ME, Xin F, Kamati KK, Cooper DN, Mooney SD, Radivojac P (2009)

Automated inference of molecular mechanisms of disease from amino acid substitutions. Bioinformatics 25:2744-50

11. Sim NL, Kumar P, Hu J, Henikoff S, Schneider G, et al. (2012) SIFT web server: predicting effects of amino acid substitutions on proteins. Nucleic Acids Res 40:W452-7

12. Adzhubei IA, Schmidt S, Peshkin L, Ramensky VE, Gerasimova A, Bork P, Kondrashov AS, Sunyaev SR (2010) A method and server for predicting damaging missense mutations. Nat Methods 7:248-9

13. Bromberg Y, Rost Burkhard (2007) SNAP: predict effect of non-synononymous polymorphisms on function. Nucleic Acids Res 35:3823-35

14. Tennessen JA, Bigham AW, O'Connor TD et al. (2012) Evolution and functional impact of rare coding variation from deep sequencing of human exomes. Science 337:64-9

15. Hwa V, Oh Y, Rosenfeld RG (1999) The insulin-like growth factor binding protein (IGFBP) superfamily. Endocrine Reviews; 20:761-787

16. Sitar T, Popowicz GM, Siwanowicz I, Huber R, Holak TA (2006) Structural basis for the inhibition of insulin-like growth factors by insulin-like growth factor-binding proteins. Proc Natl Acad Sci USA 103:13028-13033

17. Buckway CK, Wilson EM, Ahlsen M, Bang P, Oh Y, Rosenfeld RG (2001) Mutation of three critical amino acids of the N-terminal domain of IGF-binding protein-3 essential for high affinity IGF binding. J Clin Endocrinol Metab 86: 4943-4950

18. Allan GJ, Flint DJ, Patel K (2001) Insulin-like growth factor axis during embryonic development. Reproduction 122:31-39

19. Zhu W, Shiojima I, Ito Y, Li Z, Ikeda H, Yoshida M, Naito AT, Nishi J, Ueno H, Umezawa A, Minamino T, Nagai T, Kikuchi A, Asashima M, Komuro I (2008) IGFBP-4 is an inhibitor of canonical Wnt signalling required for cardiogenesis. Nature 454:345-9. 


\section{Figure legends:}

\section{Fig. 1 Genetic data of the $p \cdot R 47 W$ patient}

a) Amino acid sequence alignment of residues 44-56 of the IGFBP domain of CCN1 demonstrating very high conservation. Mutated codon 47 is shaded. The "IGFBP motif" (GCGCCxxC) is boxed. Residues that are not conserved are shown in bold.

b) Electropherogram of the heterozygous missense mutation $c .139 C>T$.

c) Localization of the detected variants (three SNPs and the $p . R 47 W$ mutation) in the CCN1 gene/protein (shown by arrows). The upper panel shows the gene structure (exons as boxes, coding sequence shaded). The lower panel shows the protein structure (SP signaling peptide, IGFBP - insulin-like growth factor binding protein domain, VWC - Von Willebrand factor domain, TSP-1 thrombospondin-homology domain, CT - cysteine knot domain, AA - amino acid).

\section{Fig. 2 Clinical vignettes of the p.R47W patient}

a) ECG demonstrating an incomplete right bundle branch block and typical crochetage pattern of the R wave in the inferior leads II, III, and aVF.

b) 2-D echocardiography revealing a large atrial septal defect (see arrowheads, modified parasternal long axis view). 
a

mutant $\mathrm{CCN} 1$

human $\mathrm{CCN} 1$

chimpanzee

treeshrew

microbat

cow

squirrel

guinea pig

tenrec

shrew

hedgehog

dog

mouse

rabbit

rat

zebrafish

human IGFBP1 human IGFBP2 human IGFBP3 human IGFBP4 b

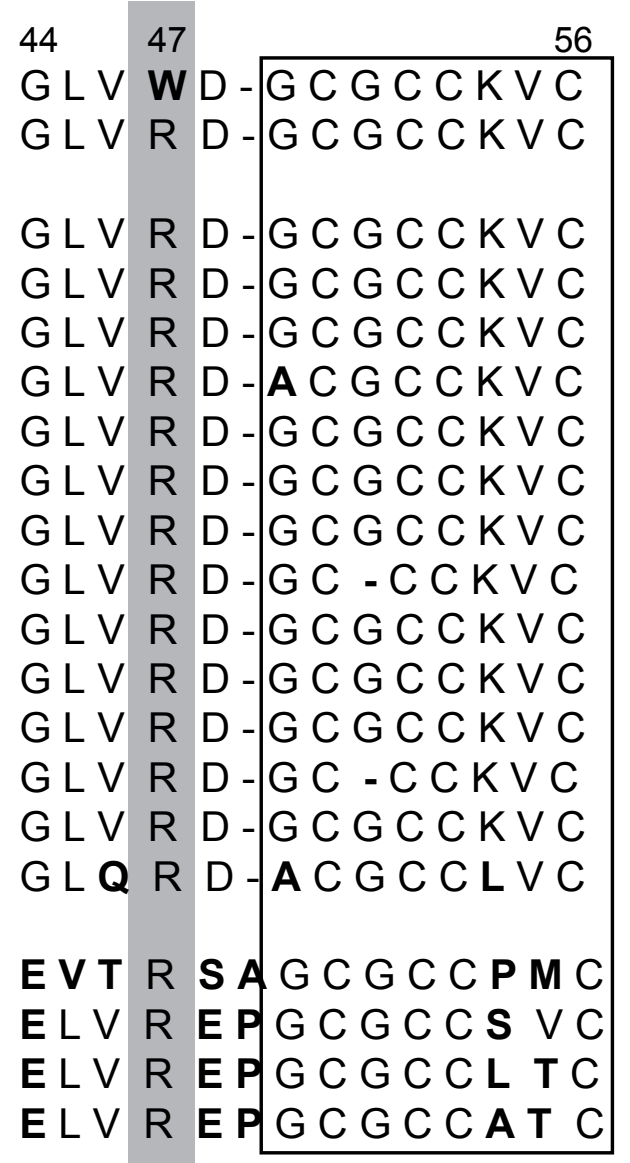

C
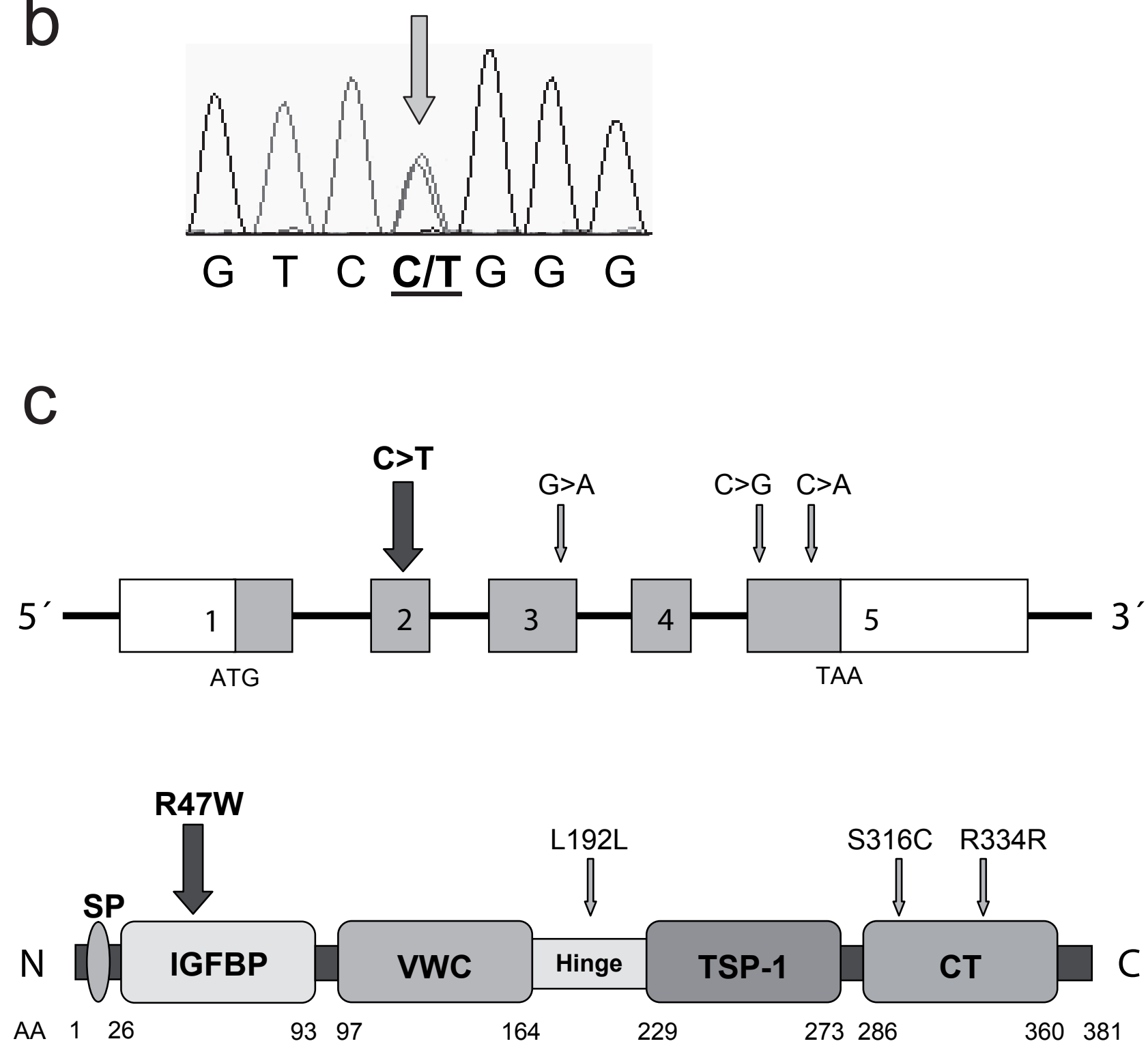
Figure 2
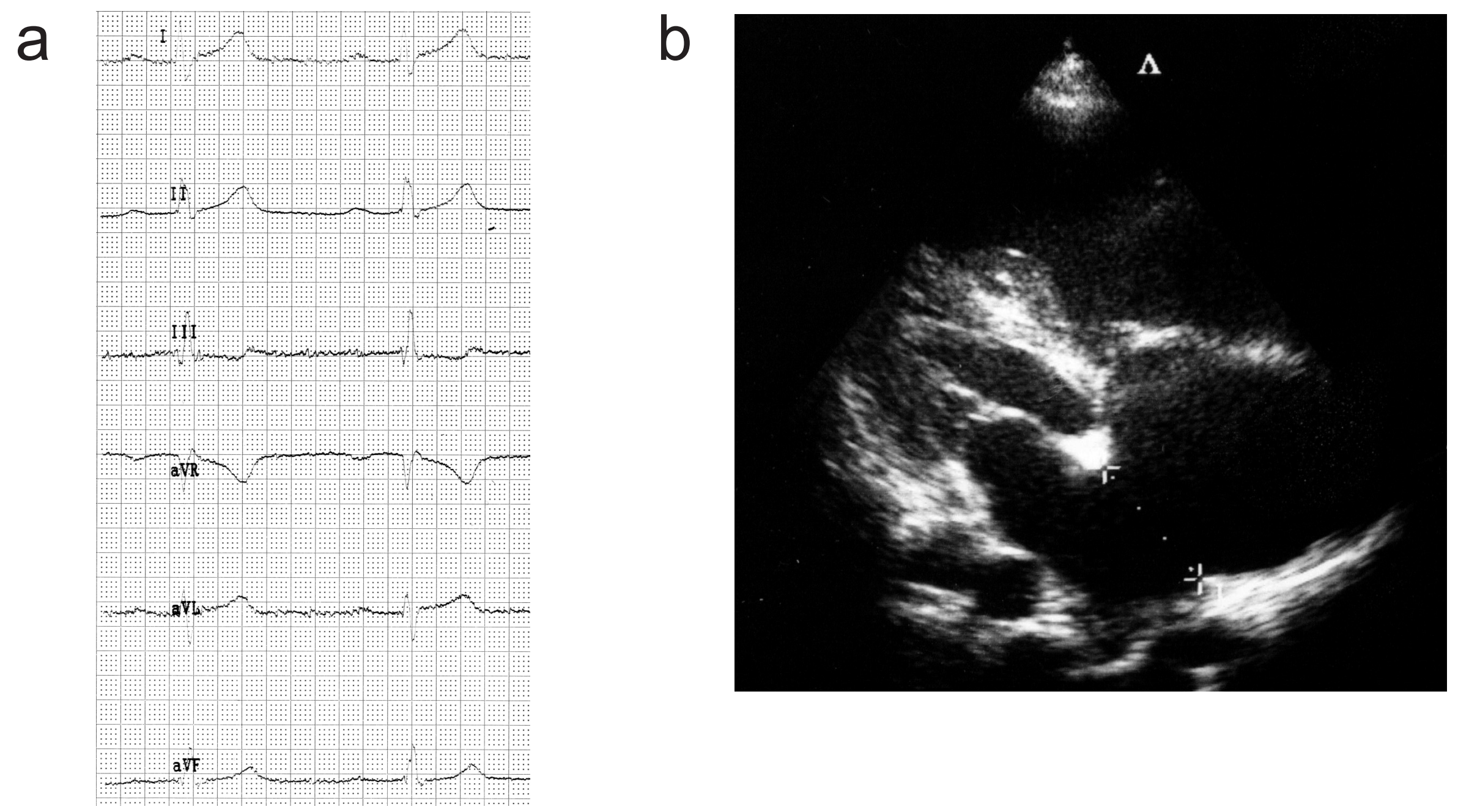